\title{
Aplicativo Pallium: Interpretando dados para o auxílio na tomada de decisões paliativas
}

\author{
Robson S. dos Santos ${ }^{1}$, Ilana de Almeida Souza Concilio ${ }^{1,2}$ \\ ${ }^{1}$ Instituto de Pesquisas Eldorado \\ Campinas - SP \\ ${ }^{2}$ Centro Universitário FIAP e Faculdades Impacta \\ São Paulo - SP \\ robsondssilva@gmail.com, ilana.concilio@eldorado.org.br
}

Abstract. Pallium is a project that was born out of a challenge with the theme of social innovation linked to its development. The product in question consists of a mobile application for $\mathrm{iOS}$ that analyzes and interprets data in order to assist the decision making of a palliative care team, providing basic, but necessary, data for the most efficient operation in any computerized hospital. The pillars of the app's design are improving the quality of life of patients, improving the workflow of palliative care teams and better management of hospital institutions' resources.

Resumo. Pallium é um projeto que nasceu de um desafio com a temática de inovação social atrelada ao seu desenvolvimento. $O$ produto em questão consiste em um aplicativo móvel para iOS que analisa e interpreta dados com o intuito de auxiliar a tomada de decisão de uma equipe de cuidados paliativos, fornecendo dados básicos, porém necessários, para o funcionamento mais eficiente em qualquer hospital informatizado. Os pilares da concepção do aplicativo são a melhora da qualidade de vida dos pacientes, melhora do fluxo de trabalho das equipes de cuidados paliativos e uma melhor gestão de recursos das instituições hospitalares.

\section{Introdução}

O IAHPC Global Project (2019) define cuidados paliativos como "cuidados holísticos ativos, ofertados a pessoas de todas as idades que encontram-se em intenso sofrimento relacionados à sua saúde, proveniente de doença grave, especialmente aquelas que estão no final da vida." [IAHPC, 2018]. É uma abordagem que visa a melhoria da qualidade de vida de pessoas com doenças graves e também em vulnerabilidade social. Segundo a Organização Mundial de Saúde (OMS), no conceito definido em 1990 e atualizado em 2002, "Cuidados paliativos consistem na assistência promovida por uma equipe multidisciplinar, que objetiva a melhoria da qualidade de vida do paciente e de seus familiares, diante de uma doença que ameace a vida, por meio da prevenção e alívio do sofrimento, da identificação precoce, avaliação impecável e tratamento da dor e demais sintomas físicos, sociais, psicológicos e espirituais." [WHO, 2007]. O termo "cuidados 
paliativos" implica uma forma personalizada de cuidados de saúde, considerando o paciente e a família ou outras pessoas importantes na unidade de cuidado e a avaliação das necessidades relacionadas à doença, sempre apoiando a qualidade de vida [Fallon and Hanks, 2006]. Segundo Souza et al (2019), as equipes multiprofissionais de saúde precisam unir esforços para conseguirem dar a assistência necessária em cuidados paliativos às pessoas e às suas famílias. Estes profissionais não podem evitar a morte ou prolongá-la, mas transformar esse processo mais tranquilo e livre de sofrimento.

Em 2015, a empresa britânica de consultoria The Economist Intelligence Unit, publicou um ranking denominado "Quality of Death Index", onde o Brasil é apontado entre as últimas posições no quesito lugar para se morrer [The Economist, 2015]. A classificação, de acordo com a publicação do próprio ranking, é relacionada com o baixo acesso ao atendimento de cuidados paliativos de qualidade e ao de medicamentos para o tratamento da dor. Segundo a Aliança Mundial de Cuidados Paliativos (Worldwide Palliative Care Alliance), mais de 20 milhões de pessoas por ano poderiam se beneficiar com o recebimento de cuidados paliativos. Entretanto, apenas $10 \%$ dos indivíduos que necessitam dessa assistência têm seu acesso garantido [Connor and Bermedo, 2014].

No Brasil, um levantamento realizado em 2018 pela Academia Nacional de Cuidados Paliativos (ANCP) apontou que, dos mais de 5 mil hospitais, dos quais cerca de metade apresentam mais de 50 leitos, apenas $10 \%$ disponibilizam uma equipe de cuidados paliativos. Ao todo existem 177 equipes no país e, dessas, mais de $50 \%$ estão concentradas na região Sudeste. Apenas $10 \%$ (cerca de 13 equipes) atendem o Norte e Nordeste. Ainda segundo o levantamento, em 2016 nos Estados Unidos já existiam mais de 1800 equipes atuando com cuidados paliativos no país, realizando a cobertura de mais de $75 \%$ dos hospitais com mais de 50 leitos [ANCP, 2018]. No Brasil, somente no dia 23 de novembro de 2018 houve, de fato, a Resolução número 41 da Comissão Intergestores Tripartite, que dispõe sobre as diretrizes para a organização deste serviço no Sistema Único de Saúde (SUS) [Ministério da Saúde, 2018].

Por conseguinte, após a interpretação das referências, dos dados coletados e contato com profissionais da área de saúde, este artigo visa apresentar a proposta e desenvolvimento do aplicativo mobile denominado Pallium. Desenvolvido em Swift (linguagem de propriedade da Apple), ele é ambientado em iOS e possui como objetivo auxiliar a rotina de trabalho dos profissionais de cuidados paliativos. Tendo em vista que esta é uma área que pode proporcionar diferenças significativas, tanto na qualidade de vida dos pacientes quanto na administração hospitalar, ela foi selecionada como foco do trabalho no âmbito da saúde. Buscou-se também fomentar no cenário brasileiro uma área da saúde que, de acordo com os dados avaliados, pode gerar retorno positivo para a sociedade em geral. 


\section{Problema}

Após confirmar os dados das pesquisas com visitas a 3 hospitais da cidade de Campinas - SP, foi definida a questão essencial do trabalho: Como identificar mais facilmente um paciente elegível para o atendimento de cuidados paliativos? Esta questão surgiu tendo em vista que, segundo os paliativistas, em geral, os pacientes não são encaminhados para os seus cuidados. Para que o trabalho paliativista ocorra, se faz necessário a realização da interconsulta, momento em que um médico de outra especialidade solicita ou sugere acompanhamento e/ou transferência do atendimento do paciente para o grupo paliativista. Todavia, isso não ocorre. Sendo necessário ao paliativista percorrer o hospital em busca de um paciente elegível ao seu atendimento. Uma pesquisa realizada por Silva et al. (2019) destaca alguns desafios relacionados aos cuidados paliativos quando realizados em domicílio como dificuldade de acesso aos serviços de apoio, questões financeiras para manutenção do profissional e dos cuidados, saúde mental e exaustão do paliativista, além da logística necessária para administração dos medicamentos.

Além de tornar o trabalho mais dinâmico, o intuito é também fornecer autonomia aos paliativistas invertendo este fluxo, uma vez que ele poderá ter em mãos dados plausíveis que podem auxiliar na proposta da interconsulta ao médico momentaneamente responsável pelo paciente.

\section{Design e Metodologia}

Para a concepção foram utilizadas algumas técnicas de design para obter uma linha de pensamento coerente que resultasse em um produto com valor real. O modelo mental Design Thinking foi utilizado para se ter um norte das ideias e insights e ter certeza de fazer um trabalho com foco nas pessoas. Os Mapas de Empatia foram decisivos para entender melhor quais as dores e necessidades dos médicos paliativistas que são o foco de uso da aplicação, e as Personas foram desenvolvidas com a função de entender melhor o segmento do mercado em que a aplicação será inserida. Aliada às técnicas supracitadas, foi utilizada a metodologia CBL (Challenge Based Learning), que possui três pilares (Engajamento, Investigação e Ação) que funcionam em um ciclo e possuem fases entremeadas que são fundamentais e devem ser sempre revisitadas [Nichols et. al, 2016].

Após essa etapa, destacaram-se algumas ferramentas de trabalho das equipes de cuidados paliativos, e que foram confirmadas com os médicos ainda na fase de investigação. Dessa forma, foi incrementado ao protótipo uma das ferramentas principais para a tomada de decisão, a escala PPS (Escala de Performance Paliativa) [Hanks, et. al, 2010]. Essa escala tem a função de auxiliar na avaliação objetiva da 
condição funcional do paciente, ela varia de $100 \%$, que significa máxima atividade funcional, até $0 \%$, indicando morte. Em qualquer uma das classificações existe a necessidade do gerenciamento intensivo dos sintomas e das demandas que o paciente e a família apresentam, [Ministério da Saúde, 2017].

\section{Desenvolvimento e Resultados}

Devido a utilização de informações críticas, foi adotado como pilar do desenvolvimento, as diretrizes da Lei Geral de Proteção aos Dados, No 13.709/18. Portanto, o aplicativo apenas interpreta e traduz as informações em sua interface gráfica, e não retém dados fora do dispositivo. Para isso, foi criado um servidor que é acessado através de um site que encaminha os dados ao aplicativo por meio de uma planilha, esta planilha é submetida somente pela equipe paliativista. Para isso, basta que o hospital possua alguma base de dados que possa emitir um arquivo em formato Excel, por exemplo.

A tela principal do aplicativo (Figura 1a) possui a lista de pacientes, que é organizada através da estrutura de cartões. Cada cartão possui as principais informações para o início das atividades dos paliativistas, são elas: Nome, idade, número de registro do paciente no hospital, tempo de internação e sua localização atual. A tela de estatísticas (Figura 1b) fornece gráficos com uma visão geral do hospital, exibindo o número de pacientes versus dias de estadia, e o número total e mensal de pacientes atendidos pela equipe de cuidados paliativos. A tela de filtro (Figura 1c) fornece modos de refinamento para exibição e dispõe de especialidades do hospital, tempo de internação e visualização de pacientes atendidos ou não atendidos.

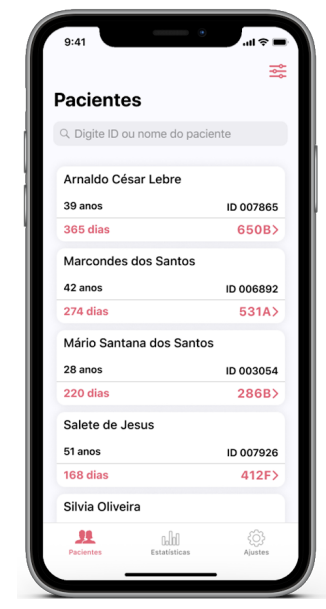

(a)

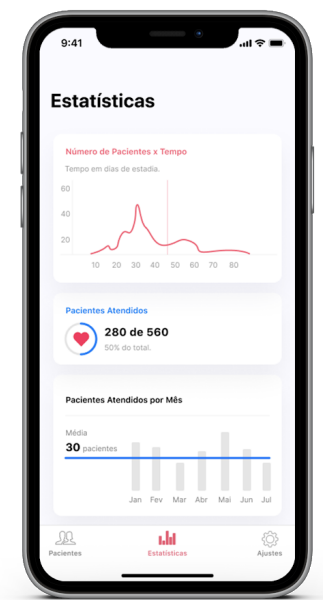

(b)

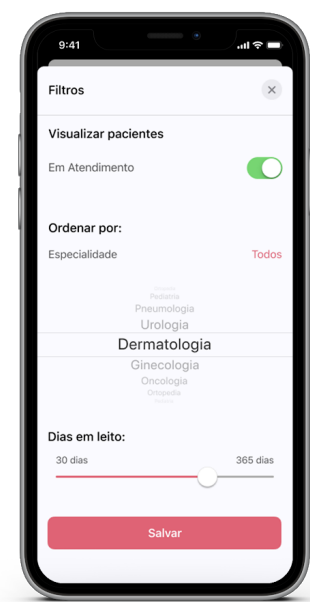

(c)

Figura 1. Telas do Pallium: (a) Principal; (b) Estatísticas e (c) Filtros.

A tela de detalhes do paciente (Figura 2a), fluxo gerado em cada card, apresenta dados mais detalhados do paciente em relação à equipe de cuidados paliativos. Além de consultar os dados, é possível ainda, na mesma tela, alterar o status do paciente para 
notificar que o atendimento pela equipe foi iniciado, adicionar observações referente ao respectivo atendimento, consultar as datas de entrada no hospital e acessar a Escala PPS. A tela de Escala PPS (Figura 2b) fornece, através de uma estrutura de lista, acesso aos fatores de cálculo da escala. O cálculo é realizado automaticamente após selecionar o valor da performance de cada fator. A tela de entradas (Figura 2c) foi projetada para fornecer em cada célula, quatro dados relacionados às entradas de cada paciente no hospital em questão: as datas de entrada e saída, a especialidade de atendimento a que fora submetido e quanto tempo o paciente permaneceu em internação, caso tenha ocorrido.

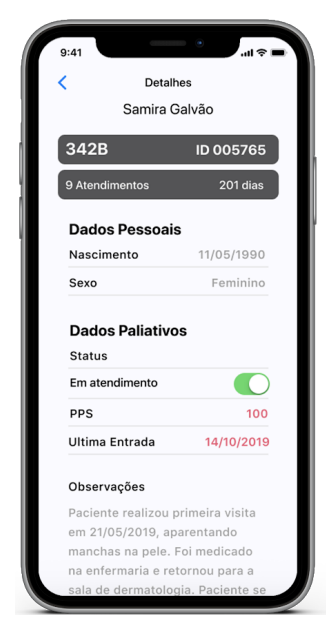

(a)

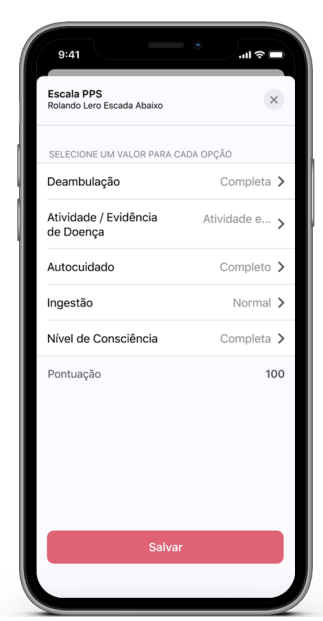

(b)



(c)

Figure 2. Telas do Pallium: (a) Detalhes do Paciente; (b) Escala PPS; e (c) Entradas do paciente.

\section{Conclusões}

Este artigo teve o objetivo de abordar a proposta do Pallium, uma aplicação idealizada a partir das problemáticas encontradas na rotina das equipes de cuidados paliativos. Apresentou informações em relação aos cuidados paliativos nos cenários nacional e internacional, e trouxe imagens do protótipo que foi desenvolvido para dispositivos móveis da plataforma iOS, com o uso da Escala PPS.

Para trabalhos futuros, pretende-se finalizar e implementar o aplicativo, analisar os resultados obtidos com e sem o uso da aplicação e implementar o uso de Machine Learning para o tratamento com grandes quantidades de dados. Além de implementar integração com bancos de dados e outras ferramentas utilizadas pelos profissionais.

\section{Agradecimentos}

Os autores agradecem aos instrutores e colegas do Apple Developer Academy do Instituto de Pesquisas Eldorado em Campinas-SP pelo apoio. Em destaque, agradecem 
também aos membros da equipe desenvolvedora do aplicativo Pallium: André Papoti de Oliveira, Hatos Albert Barbosa e João Pedro de Amorim.

\section{Referências}

ANCP, (2018) "Panorama dos Cuidados Paliativos no Brasil", https://paliativo.org.br/wp-content/uploads/2018/10/Panorama-dos-Cuidados-Paliativ os-no-Brasil-2018.pdf. Acessado em Março de 2020.

Connor, S. R. and Bermedo, M. C. S. (2014) "Global Atlas of Palliative Care at the End of Life", https://www.who.int/nmh/Global Atlas of Palliative Care.pdf, Acessado em Março de 2020.

Fallon, M. and Hanks, G. (2006) “ABC of Palliative Care”, 2st ed.

Hanks, G., Cherny, I. N., Christakis, A. N., Fallon, M., Kaasa, S. and Portenoy, K. R., (2010) "Oxford Textbook of Palliative Care", 4th ed.

IAHPC - International Association for Hospice and Palliative Care, (2018) "Palliative Care Definition",https://hospicecare.com/what-we-do/projects/consensus-baseddefinition-of-palliative-care/definition/. Acessado em Março de 2020.

Ministério da Saúde, (2017) "Cuidados paliativos", Livro Digital. https://telessaude.hc.ufmg.br/wp-content/uploads/2016/07/CUIDADOS-PALIATIV OS LIVRO.pdf. Acessado em Março de 2020.

Ministério da Saúde, (2018) "Resolução no 41, de 31 de Outubro de 2018", https://portalarquivos2.saude.gov.br/images/pdf/2018/novembro/23/RESOLUCAON41.pdf. Acessado em Março de 2020.

Nichols, M., Cator, K., and Torres, M. (2016) Challenge Based Learner User Guide. Redwood City, CA: Digital Promise.

Silva, A.E., Braga, P.P., Sena, R.R., Duarte, E.D. and Sena, L.R. (2019) Cuidados Paliativos Domiciliares: Revisão Integrativa. Ciência, Cuidado E Saúde, 18(3). https://doi.org/10.4025/cienccuidsaude.v18i3.41994.

Souza, D. L. S., Braga, K. L., Silva, M. L. and Quental, O. B. (2019) Conhecer A Percepção Da Equipe Médica Sobre Cuidados Paliativos: Revisão Integrativa. Id on Line Revista Multidisciplinar e de Psicologia. V. 13, N. 48 p. 831-838, Dezembro/2019. DOI: https://doi.org/10.14295/idonline.v13i48.2293

The Economist, (2015) "The 2015 Quality of Death Index Ranking palliative care across the world", https://eiuperspectives.economist.com/sites/default/files/ 2015\%20EIU\%20Quality\%20of\%20Death\%20Index\%200ct\%2029\%20FINAL.pdf. Acessado em Março de 2020.

WHO - World Health Organization, (2007) "Cancer Control: Knowledge into Action WHO Guide for "Cffective Programmes", https://www.who.int/cancer/media/FINAL-PalliativeCareModule.pdf. Acessado em Março de 2020. 